\section{Superficial Siderosis in Cerebral Amyloid Angiopathy}

With great interest, we read the recent review article by Kumar entitled "Neuroimaging in Superficial Siderosis: An In-Depth Look," published on-line ahead of print in the American Journal of Neuroradiology in September 2009. ${ }^{1}$ The author provides a thorough review of neuroimaging in superficial siderosis (SS) of the central nervous system and details important underlying causes of this phenomenon. Besides the common mechanisms (eg, history of trauma or intradural surgery), the author lists cerebral amyloid angiopathy (CAA) as a potential pathomechanism for SS. ${ }^{1}$ We want to underline the role of this microangiopathic disease as an important cause of SS.

Most interesting, the published CAA cases with SS lack the typical clinical findings of "classic" SS, which are progressive gait ataxia with cerebellar dysarthria and sensorineural hearing loss, but patients often present with headache, seizures, and cognitive impairment. ${ }^{2-4}$ This is most probably due to the characteristic localization of SS in patients with CAA: While the "classic" SS mainly affects brain stem and posterior fossa, ${ }^{1} \mathrm{SS}$ in CAA is typically found in a supratentorial distribution over the cerebral convexities. ${ }^{2-4}$

In our opinion, CAA should be thoroughly considered as a cause of SS, especially in older patients with isolated supratentorial SS and an atypical clinical presentation. Further studies on the sensitivity and specificity of SS as a noninvasive diagnostic MR imaging sign of CAA are necessary.

\section{References}

1. Kumar N. Neuroimaging in superficial siderosis: an in-depth look. AJNR AmJ Neuroradiol 2010;31:5-14

2. Linn J, Herms J, Dichgans M, et al. Subarachnoid hemosiderosis and superficial cortical hemosiderosis in cerebral amyloid angiopathy. AJNR Am J Neuroradiol 2008;29:184-86

3. Roch JA, Nighoghossian N, Hermier M, et al. Transient neurologic symptoms related to cerebral amyloid angiopathy: usefulness of $\mathrm{T} 2{ }^{*}$-weighted imaging. Cerebrovasc Dis 2005;20:412-14

4. Feldman HH, Maia LF, Mackenzie IR, et al. Superficial siderosis: a potential diagnostic marker of cerebral amyloid angiopathy in Alzheimer disease. Stroke 2008;39:2894-97

J. Linn

H. Brückmann

Department of Neuroradiology University Hospital Munich Munich, Germany

DOI 10.3174/ajnr.A1913 